\title{
Level set based method for design of compliant mechanisms
}

\author{
François Jouve $^{(1)}$, Houari Mechkour ${ }^{(2)}$ \\ (1) Laboratoire J.L. Lions (UMR 7598) \\ Université Paris Diderot \\ 75252 Paris Cedex 05, France \\ jouve@math.jussieu.fr \\ (2) Centre de Mathématiques Appliquées (UMR 7641) \\ École Polytechnique, 91128 Palaiseau, France \\ mechkour@cmap.polytechnique.fr
}

\begin{abstract}
In the context of structural optimization, we propose two natural extensions of the level set method for the design of compliant mechanisms. Two new objective functions are introduced, well suited to the automatic design of compliant mechanisms, and a strategy for the design of mechanisms adapted to multiple loads is proposed. This work is illustrated with several numericals examples.

RÉSUMÉ. Dans le cadre de l'optimisation de structures élastiques, nous proposons deux extensions naturelles de la méthode des lignes de niveaux qui peuvent s'avérer utiles aux concepteurs de micro-mécanismes. Nous introduisons une nouvelle fonction-coût, inspirée d'un critère d'optimisation largement utilisé dans le domaine de la conception de mécanismes, ainsi qu'une stratégie pour traiter le cas de mécanismes adaptés à plusieurs chargements (multichargement). Ce travail est illustré de quelques exemples numériques.

KEYWORDS: Shape optimization, Compliant mecanisms, Level set.

MOTS-CLÉS : Optimisation de forme, Mécanismes compliants, Courbes de niveau.
\end{abstract}

REMN - 17/2008. Giens 2007, pages 957 à 971 


\section{Introduction}

A compliant mechanism is a single-body structure, which uses its elastic deformations to achieve a given action. Wide range of uses for compliant mechanisms include smart structures, semi-invasive medical surgical devices and micro-electromechanical-systems (MEMS).

There are essentially two classes of numerical methods for the design of compliant mechanisms: the kinematic synthesis approach based on the traditional rigid-body kinematics ((Howell et al.,, 1994), (Howell,, 2001)) and the topology optimization method for continuum structures ((Ananthasuresh et al.,, 1994), (Frecker et al.,, 1997), (Sigmund,, 1997)). The method proposed in this work belongs to the second category. It is based on the level set method.

In the late 80's, Osher and Sethian introduced the level set representation for numerically tracking fronts and free boundaries (Osher et al.,, 1988). It is now widely used in various applications. The level set method has recently been introduced in the topology and shape optimization field (see (Allaire et al.,, 2002), (Allaire et al.,, 2004), (Allaire et al.,, 2005c), (Wang et al.,, 2003), (Wang et al.,, 2005)) and has become very popular because of its ability to handle arbitrary objective functions and state equations, and also for its great efficiency and versatility.

Basically, the level set method revisits the classical shape optimization techniques - based on the shape derivative - using the level set representation on a fixed mesh. It avoids most of the flaws of the classical gradient methods: the numerical instabilities and the computational difficulties related to the remeshing of the shapes at each step of the boundary variation algorithms are cancelled. Moreover, the level set method is very easy to implement en $3 \mathrm{~d}$. But the well known advantages of the classical methods are preserved: the capability to use almost any constitutive equation and any objective function.

One remaining flaw, still present in the genuine level set method, is the possibility of the algorithm to be "trapped" into local minima and to be unable to find the "good" topology, starting from any initial shape: for this reason, the method is frequently described as not being a fully topological method, and as very sensitive to initial guesses (which is actually almost the same argument). This remark is mathematically founded, but not completely fair from a numerical point of view: our numerical experience shows that, as soon as the initial structure is "rich enough" topologically (i.e. there are enough initial holes), the solutions obtained do not depend on initialization. Moreover, it has been shown in (Allaire et al.,, 2005b) that the coupling with the topological gradient is very effective, making these critics almost irrelevant.

In this paper we concentrate on the new objective function introduced to compute optimal compliant mechanisms, and we will not discuss some details of the method that can be found in the references cited. The method is described in the framework of linearized elasticity, although it is obvious that large deformations are likely to occur in actual compliant mechanisms, and nonlinear elasticity should be used in real 
applications. Just remember that the method presented here can be easily extended to nonlinear models (cf. (Allaire et al.,, 2004)), as soon as efficient solvers for the direct problem are available.

Compared to the basic level set method proposed in (Allaire et al.,, 2004), we add two significant extensions, quite natural to introduce, but that could be very useful to the actual conception of compliant mechanisms:

1) new cost functions: we introduce a new objective functions in variational form that is well suited to the computation of the shape derivative that is to be computed in our algorithm. It can be compared to a widely used objective function, usually written in a vector or discrete form: the mechanical advantage (MA) which is maximizing the ratio between the output and input forces. Our integral formulation can handle, without modifications, multiple input and output ports;

2) multi-loads: We show that our formulation can deal with multiple loads cases, where the same structure is designed to be able to reach a given target displacement through different input ports and forces.

\section{Analysis of optimal synthesis of compliant mechanisms}

\subsection{Setting of the problem}

Let $\Omega \subset \mathbb{R}^{d}$ ( $d=2$ or 3 ) be a bounded open set occupied by a linear isotropic elastic material with Hooke's law denoted by $A$. The boundary of $\Omega$ is made of two disjoint parts $\partial \Omega=\Gamma_{N} \cup \Gamma_{D}$, with Dirichlet boundary conditions imposed on $\Gamma_{D}$, and Neumann boundary conditions on $\Gamma_{N}$. All admissible shapes $\Omega$ are required to be a subset of a working domain $D \subset \mathbb{R}^{d}$. We denote by $f$ the vector-valued function of the volume forces and by $g$ that of the surface loads. The displacement field $u$ in $\Omega$ is the solution of the linearized elasticity system

$$
\left\{\begin{aligned}
-\operatorname{div}(A e(u)) & =f & & \text { in } \Omega, \\
u & =0 & & \text { on } \Gamma_{D}, \\
(A e(u)) n & =g & & \text { on } \Gamma_{N},
\end{aligned}\right.
$$

where $e(u)=\frac{1}{2}\left(\nabla u+\nabla u^{T}\right)$ is the strain tensor.

The shape optimization problem is formulated as a minimization problem inf $J(\Omega)$, where $J(\Omega)$ is an objective function chosen to evaluate the perfor$\Omega$ admissible mance of the mechanism. In the context of compliant mechanism optimization, many fitness functions are useful, that lead to different optimal designs. The most used in the literature are available in our numerical implementation of the level set method: mechanical advantage (MA), geometrical advantage (GA), work ratio (WR) (or mechanical efficiency ME) and a least square error criterion (for details, see (Allaire $e t$ al.,, 2004), (Allaire et al.,, 2005a), (Wang et al.,, 2005), (Jouve et al.,, 2007)). Only one of them will be described bellow. 
The basic problem in compliant mechanism design consists in synthesizing a mechanism topology that transfers an input to a desired mechanical output in an energy-efficient way. There are two design objectives to be met simultaneously when designing a compliant mechanisms: (i) flexible enough to satisfy the kinematic requirements and (ii) stiff enough to support external loads. These design objectives are usually antagonist. The input to the compliant mechanism can be a prescribed force or a prescribed displacement. In this paper, the input is assumed to be a prescribed force $F_{i n}=\left\|\vec{F}_{i n}\right\|$.

Figure 1 illustrates a schematic monolithic compliant mechanism, with a fixed boundary subset $\Gamma_{D}$. At the input port $\omega_{i n}$, an input force $F_{i n}$ is applied, while the displacement magnitude $u_{\text {out }}$ at the output port $\omega_{\text {out }}$ is computed and projected onto a given direction indicated by the vector $l_{\text {out }}$. The displacement over the output port $\omega_{\text {out }}$ in a specified direction $l_{\text {out }}$ can be used as a measure for the intended flexibility in a compliant mechanism (see (Saxena et al.,, 2000)).

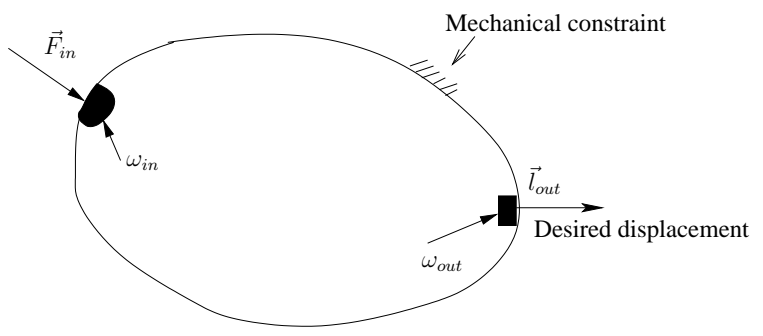

Figure 1. A schematic monolithic compliant mechanisms with input force and output displacement

In this paper we illustrate our approach with one particular objective function, that can be related to the Geometrical Advantage (GA) classically used in the literature. The main difference lays in the "continuous" formulation, necessary for the computation of the shape derivative. The objective is to obtain a structure that maximises the displacement at the output port in a specified direction. This can be formulated by the following minimization problem

$$
J(\Omega)=-\frac{1}{\left|\omega_{\text {out }}\right|} \int_{\Omega} \chi_{\text {out }}(x)\left(l_{\text {out }}(x), u(x)\right) d x
$$

where $\chi_{\text {out }}$ is the characteristic function associated to the output port(s).

\subsection{Sensitivity analysis using the shape derivative}

The level set method is mixing two basic ingredients: the classical shape sensitivity analysis and the level set representation. To find a solution to the optimization 
problem, we need to find the variation of the objective function with respect to a variation of the design variables. The sensitivity of boundary perturbations is measured by the shape derivative. In the litterature, there exist many pioneering work on shape sensitivity analysis. In the present study, the shape sensitivity analysis detailed in (Céa,, 1986)(Allaire et al.,, 2002) is adopted to derive the shape derivatives by using the well-known approach of Murat and Simon (see (Murat et al.,, 1976)).

After computation we find the expression for the shape derivative of the objective function (2), measuring the variations of $J$ with respect to infinitesimally small variations of the boundary $\partial \Omega$ of $\Omega$ characterized by a vector field $\theta$ (the boundary of $\partial \Omega$ being assumed to be smooth enough):

$$
\begin{aligned}
J^{\prime}(\Omega)(\theta) & =\int_{\partial \Omega}\left(\frac{1}{\left|\omega_{\text {out }}\right|}\left(l_{\text {out }}, \chi_{\text {out }} u\right)+A e(p) \cdot e(u)\right) \theta \cdot n \mathrm{~d} s \\
& -\int_{\Gamma_{N}}\left(f \cdot p+\frac{\partial(g \cdot p)}{\partial n}+H g \cdot p\right) \theta \cdot n \mathrm{~d} s
\end{aligned}
$$

where $n$ and $H$ are respectively the exterior normal vector and the mean curvature, and $p$ is the adjoint state, supposed to be smooth, i.e. $p \in H^{2}(\Omega)^{d}$, defined as the solution of the adjoint problem

$$
\begin{cases}-\operatorname{div}(A e(p))=\frac{1}{\left|\omega_{\text {out }}\right|} \chi_{\text {out }} l_{\text {out }} & \text { on } \Omega \\ p=0 & \text { in } \Gamma_{D} \\ (A e(p)) n=0 & \text { in } \Gamma_{N}\end{cases}
$$

\subsection{Shape representation by level set method}

In the level set method, the boundary of a solid structure is not explicitly computed, but rather implicitly represented as the zero level set of a scalar field $\psi(x)$, defined over the whole reference domain $D$, which is called the level set function. If the solid region is $\Omega \subset D$, then, following the idea of Osher and Sethian (Osher et al.,, 1988), $\Omega$ and $\partial \Omega$ are implicitly described through the zero level set of $\psi$ by

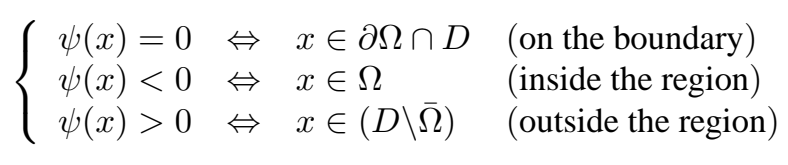

The outward unit vector $n$ normal to the boundary $\partial \Omega$ is obtained by $n=-\nabla \psi /|\nabla \psi|$ and the mean curvature $H$ is given by $\operatorname{div}(\nabla \psi /|\nabla \psi|)$. During the optimization process, the shape $\Omega(t)$ is going to evolve according to a pseudo time parameter $t \in \mathbb{R}^{+}$, which corresponds to the down stepping parameter of a classical gradient method. 
Doing variations of the shape in the direction of the shape gradient amounts to solve the following Hamilton-Jacobi transport equation (see. (Osher et al.,, 1988), (Allaire et al., , 2004) for details),

$$
\frac{\partial \psi}{\partial t}+V|\nabla \psi|=0 \text { in } D, \frac{\partial \psi}{\partial n}=0 \text { in } \partial D
$$

where $V(x, t)$ is the normal velocity of the shape's boundary, computed using the shape derivative given by formula (3).

The basic idea of the method is to avoid the actual computation of the zero level set and the explicit description of the domain $\Omega$, allowing to make all the computations using the same mesh of the whole computational domain $D$. In this spirit, the elasticity system governing the state $u$ (as well as the adjoint state $p$ ) is extended to the whole domain $D$ using the so-called "ersatz material" approach. More precisely we define an elasticity tensor $A^{*}(x)$ over the whole domain $D$. $A^{*}$ is equal to $A$ in $\Omega$ and to the weak material $\varepsilon A$ in $D \backslash \bar{\Omega}$, mimicking holes. The constant $\varepsilon$ must be chosen small enough, such that the problem (1) is approximated with a good accuracy, but not too small to avoid numerical instabilities.

\subsection{Numerical algorithm}

We use the level set algorithm, proposed in (Allaire et al.,, 2002)(Allaire et al.,, 2004). It is an iterative algorithm, structured as follows:

1) initialization of the level set function $\psi_{0}$ corresponding to an initial guess $\Omega_{0}$. Typically, $\Omega_{0}$ is the full domain $D$ perforated by a periodic distribution of circular holes;

2) iteration until convergence, for $k \geq 0$ :

a) computation of the state $u_{k}$ and the adjoint state $p_{k}$ through two linear elasticity problems (1) posed in $\Omega_{k}$;

b) deformation of the shape by solving the transport Hamilton-Jacobi equation (6). The new shape $\Omega_{k+1}$ is characterized by the level set function $\psi_{k+1}$ solution of (6) after a time step $\Delta t_{k}$ starting from the initial condition $\psi_{k}(x)$ with velocity $V_{k}=-v_{k}$ computed in terms of $u_{k}$ and $p_{k}$. The time step $\Delta t_{k}$ is chosen such that $J\left(\Omega_{k+1}\right) \leq J\left(\Omega_{k}\right)$

3) from time to time, for stability reasons, we also reinitialize the level set function $\psi$ by solving

$$
\left\{\begin{array}{cl}
\frac{\partial \psi_{k}}{\partial t}+\operatorname{sign}\left(\psi_{k-1}\right)\left(\left|\nabla \psi_{k}\right|-1\right)=0 & \text { in } D \times \mathbb{R}^{+} \\
\psi_{k}(t=0, x)=\psi_{k-1}(x) & \text { in } D
\end{array}\right.
$$

which admits the signed distance to the zero level set as a stationary solution. 
The Hamilton-Jacobi equation (6) is solved by an explicit upwind scheme on a Cartesian grid with a time stepping satisfying a CFL condition (see e.g. (Sethian et al.,, 2000) and (Allaire et al.,, 2004) for details).

\section{Numerical results}

The numerical examples presented in this section address three different issues:

1) multiple inputs or outputs ports,

2) multiple loadings,

3) three dimensional case.

In all these computations, it is assumed that the constitutive material has a Young's modulus $E_{0}=1$ and the weak material has a Young's modulus $E_{1}=\varepsilon E_{0}$, with $\varepsilon=10^{-2}$. The Poisson's ratio is $\nu=0.3$ in both phases. Remark that the actual value of the Young's modulus $E_{0}$ is not relevant since our model is the linear elasticity.

\subsection{Multiple input and outputs ports cases}

The following examples demonstrate the applicability of the method to treat multiple input/output ports problems.

\subsubsection{Classical force inverter}

The first case is a very classical test, described for example in (Sigmund,, 1997). An horizontal force is applied on the input zone and we are looking for a maximal displacement on the output port in the opposite direction. The mechanism is allowed be clamped on two zones on the left edge where a Dirichlet boundary condition is imposed (cf. Figure 2a). The optimal solution and its deformed state are presented on Figures 2b-c.

(a)

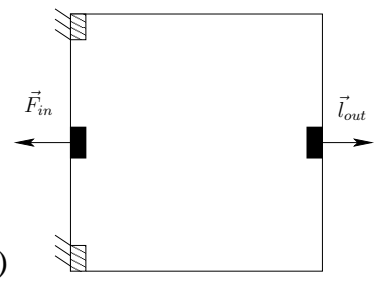

(b)

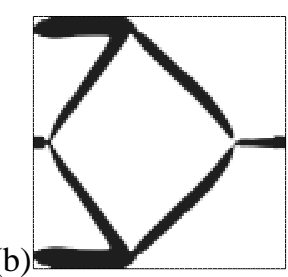

(c)

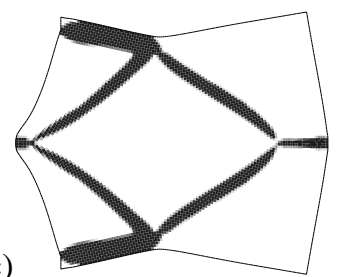

Figure 2. Force inverter. (a): design domain and specifications of the problem, $(b)$ : optimal solution, (c): deformed configuration 


\subsubsection{Multiple output ports}

The next example shares the same reference domain, boundary conditions and input port with the previous one. The direction of the force is in the opposite direction. Two output zones are defined and the target displacement is shown on Figure 3 a. The final shape of this force inverter and its deformed shape are presented on Figures $3 \mathrm{~b}$-c.

(a)

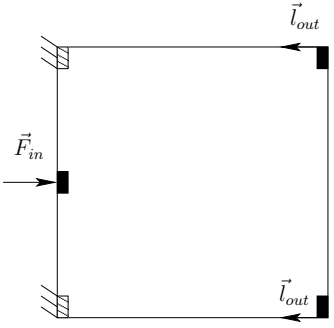

(b)

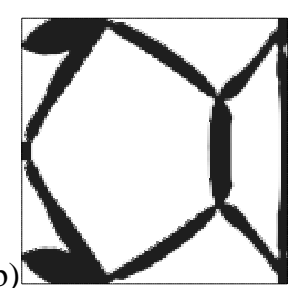

(c)

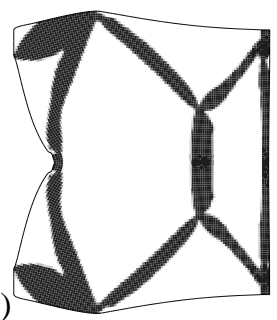

Figure 3. Force inverter with two output ports. (a): design domain and specifications of the problem, $(b)$ : optimal solution, $(c)$ : deformed configuration

An important remark can be done about the Dirichlet boundary conditions. They are imposed on a fixed part $\Gamma_{D}$ of the boundary of $D$, but the final optimized structure has no reasons to include entirely $\Gamma_{D}$. If a better solution can be found with only a part of Gamma $_{D}$ included in $\partial \Omega$, the algorithm can find it. The two following examples illustrate this behaviour.

The first example has the same specifications than the previous one (Figure 3), but $\Gamma_{D}$ is now wider, covering almost the whole boundary of $D$. Figure 4 shows the optimized solution. Notice that only a small part of $\Gamma_{D}$ belongs to the boundary of the optimal structure.

(a)

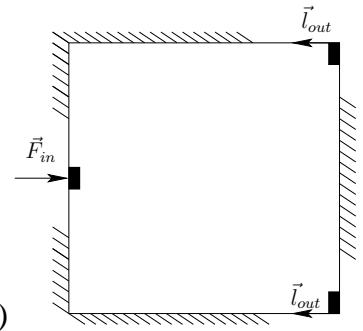

(b)

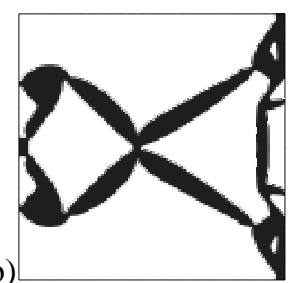

(c)

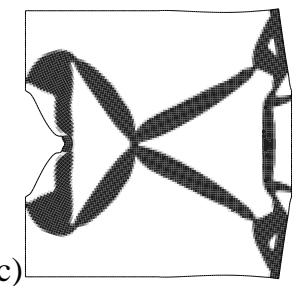

Figure 4. Force inverter with two output ports and more flexibility on the choice of the location of the Dirichlet boundary conditions. (a): design domain and specifications of the problem, $(b)$ : optimal solution, $(c)$ : deformed configuration

The last example of this section has the same input/output ports and boundary conditions than Figure 4 but the target displacement has different directions on the two 
output ports (Figure 5a). The expected structure has no reason to be symmetric, and the computed solution is in fact non-symmetric (Figure 5b). This example demonstrates the ability of the algorithm to find complex topologies and solutions far from the common mechanical intuition.

(a)

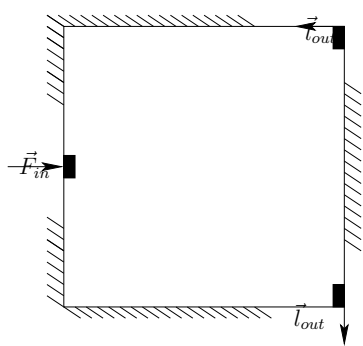

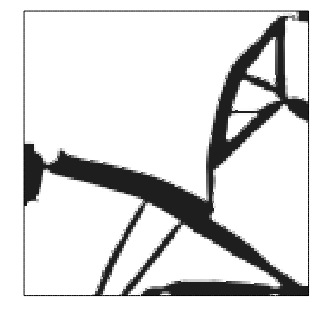

(b)

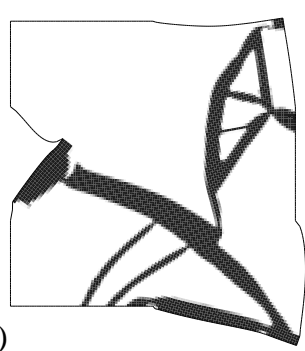

(c)

Figure 5. Force inverter with two output ports. (a): design domain and specifications of the problem, $(b)$ : optimal solution, $(c)$ : deformed configuration

\subsection{Single and multiple loads case}

The method can also handle multiple loads cases, where a single structure is optimal for different external forces. In the example bellow, three loadings are defined. The solution has to be optimal for each of the loading case (see (Allaire et al.,, 2005c) for details in an other context). The design configuration and the loadings are shown on Figure 6. The optimal solution and its deformed configuration for each of the three loading cases are displayed on Figures $7 \mathrm{a}-\mathrm{d}$.

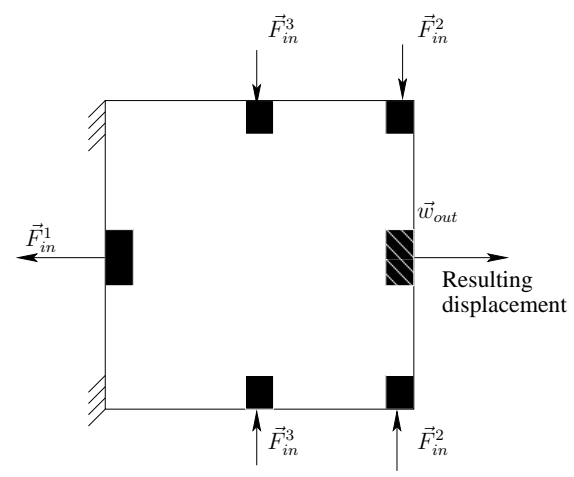

Figure 6. Design problem specifications for the multiple loads force inverter 


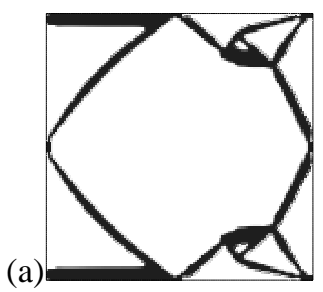

(b)

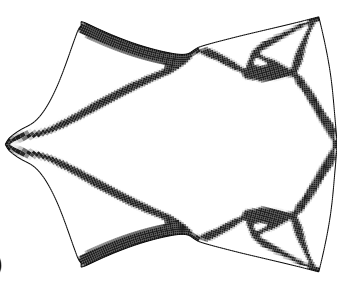

(c)

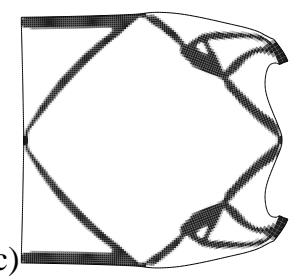

(d)

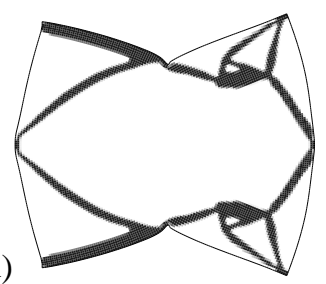

Figure 7. Force inverter with three loads: (a): final design, $(b)$ : deformed configuration for the first loading, (c): deformed configuration for the second loading, $(d)$ : deformed configuration for the third loading

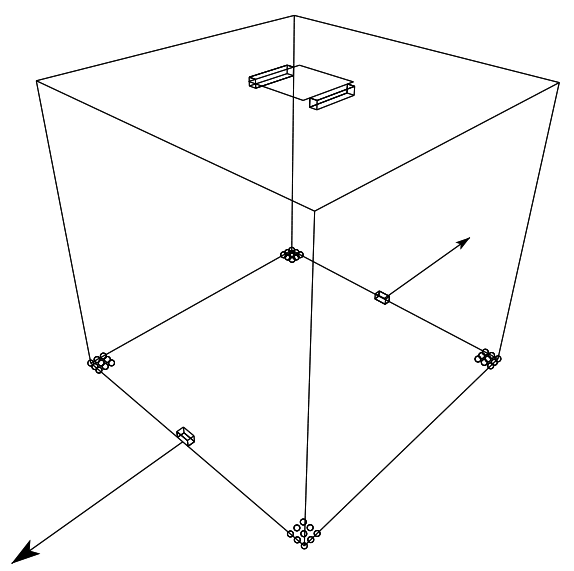

Figure 8. Three-dimensional micro-gripper: applied forces and boundary conditions (circles on the nodes submitted to Dirichlet boundary conditions) 


\subsection{Three dimensional case}

The method can be applied to three-dimensional cases with no modifications. The implicit representation of the shapes is particularly adapted to the $3 \mathrm{~d}$ problems since it avoid remeshing.

As an example we have optimized the tridimensional gripper presented on Figure 9. The design domain is a parallelepiped of dimension $0.5 \times 0.5 \times 1$. Due to symmetries, the computation can be performed on a quarter of the domain, reducing the computational cost. The gripper is clamped at its 4 lower corners and is pulled horizontally and symmetrically on 2 small zones in the middle of 2 edges of the lower face (see Figure 8). The objective function (i.e. $\omega_{\text {out }}$ and $\ell_{\text {out }}$ ) is chosen to allow the jaws (situated in the middle of the top face of the cube) to close when the forces are applied.
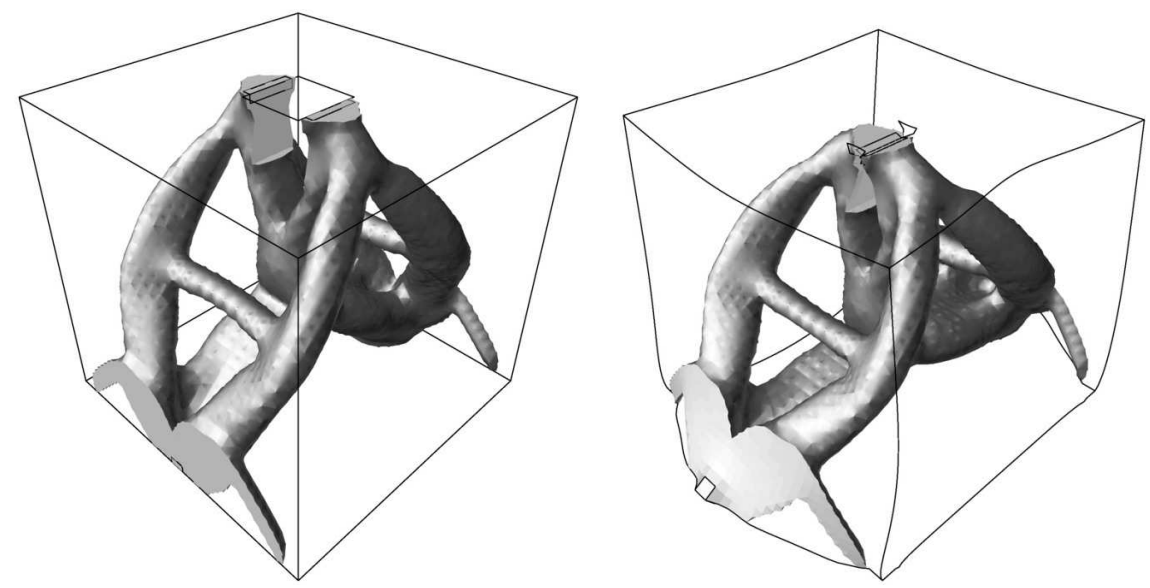

Figure 9. Three-dimensional micro-gripper: optimized design and deformed configuration

\section{References}

Allaire G., De Gournay F., Jouve F., « Optimisation de forme de micro-mécanismes compliants par la méthode des courbes de niveau », Actes du 7ème Colloque National en Calcul des Structures, Hermes, Giens, p. 229-234, 2005a.

Allaire G., De Gournay F., Jouve F., Toader A., « Structural optimization using topological and shape sensitivity via a level set method », Control and Cybernetics, vol. 34, p. 59-80, 2005 b.

Allaire G., Jouve F., « A level-set method for vibration and multiple loads structural optimization », Comput. Methods Appl. Mech. Engrg., vol. 194, p. 3269-3290, 2005c.

Allaire G., Jouve F., Toader A.-M., « A level-set method for shape optimization », C. R. Acad. Sci., Paris, Série I, vol. 334, p. 1125-1130, 2002. 
Allaire G., Jouve F., Toader A.-M., « Structural optimization using sensitivity analysis and a level-set method », J. Comp. Phys.,, vol. 194, n 1, p. 363-393, 2004.

Ananthasuresh G., Kota S., Gianchandani Y., « A methodical approach to design of compliant micro-mechanisms », In Solid-State Sensor and Actuator Workshop, p. 189-192, 1994.

Céa J., « Conception optimale ou identification de formes, calcul rapide de la dérivée directionnelle de la fonction coût », Math. Model. Num. Anal., vol. 20, n 3, p. 371-402, 1986.

Frecker M., Ananthasuresh G., Nishiwaki S., Kikuchi N., « Topology synthesis of compliant mechanisms using multi-criteria optimization », J. Mech. Des. Trans ASME, vol. 119, n² 2 , p. 238-245, 1997.

Howell L., Compliant mechanisms, John Wiley \& Sons, New York, 2001.

Howell L., Midha A., «A method for design of compliant mechanisms with small length flexural pivots », ASME J. Mech. Design, vol. 116, p. 280-290, 1994.

Jouve F., Mechkour H., « Optimization assisted design of compliant mechanisms by the level set method », In Proc. of 12th World Congress in Mechanism and Machine Science, IFToMM, Besançon, France, June 18-21, 2007.

Murat F., Simon J., « Etudes de problèmes d'optimal design », Lectures Notes in Computer Science 41, Springer Verlag, Berlin, p. 54-62, 1976.

Osher S., Sethian J., « Front propagating with curvature dependent speed: algorithms based on Hamilton-Jacobi formulations », J. Comp. Phys., vol. 78, p. 12-49, 1988.

Saxena A., Ananthasuresh G., « On an optimal property of compliant topologies », Struct Multidisc Optim., vol. 19, p. 36-49, 2000.

Sethian J., Wiegmann A., « Structural boundary design via level set and immersed interface methods », J. Comp. Phys., vol. 163, p. 489-528, 2000.

Sigmund O., « On the design of compliant mechanisms using topology optimization », Mech. Struct. Mach., vol. 25, p. 493-524, 1997.

Wang M., Chen S., Wang X., Mei Y., « Design of multimaterial compliant mechanisms using level set methods », ASME J. Mech. Design, vol. 127, p. 941-955, 2005.

Wang M., Wang D., Guo A., « A level set method for structural topology optimization », Comput. Methods Appl. Engrg., vol. 192, p. 227-246, 2003. 


\section{ANNEXE POUR LE SERVICE FABRICATION \\ A FOURNIR PAR LES AUTEURS AVEC UN EXEMPLAIRE PAPIER \\ DE LEUR ARTICLE ET LE COPYRIGHT SIGNE PAR COURRIER \\ LE FICHIER PDF CORRESPONDANT SERA ENVOYE PAR E-MAIL}

1. ARTICLE POUR LA REVUE :

REMN - 17/2008. Giens 2007

2. Auteurs :

François Jouve $^{(1)}$, Houari Mechkour ${ }^{(2)}$

3. TITRE DE L'ARTICLE :

Level set based method for design of compliant mechanisms

4. Titre ABRÉGÉ POUR LE haUT DE PAGE MOINS DE 40 Signes :

Compliant mechanisms design by level set

5. DATE DE CETTE VERSiON :

July 2, 2008

6. CoOrdonnées des Auteurs :

- adresse postale :

(1) Laboratoire J.L. Lions (UMR 7598)

Université Paris Diderot

75252 Paris Cedex 05, France

jouve@math.jussieu.fr

(2) Centre de Mathématiques Appliquées (UMR 7641)

École Polytechnique, 91128 Palaiseau, France

mechkour@cmap.polytechnique.fr

- téléphone : 0144276967

- télécopie : 0169333011

- e-mail : jouve@math.jussieu.fr

7. LOGICIEL UTILISÉ POUR LA PRÉPARATION DE CET ARTICLE :

$\mathrm{HT}_{\mathrm{E}} \mathrm{X}$, avec le fichier de style article-hermes.cls, version $1.23 \mathrm{du} 17 / 11 / 2005$.

8. FORMULAIRE DE COPYRIGHT :

Retourner le formulaire de copyright signé par les auteurs, téléchargé sur :

http: //www.revuesonline.com

SERVICE ÉDITORIAL - HERMES-LAVOISIER 14 rue de Provigny, F-94236 Cachan cedex 